\title{
Velocidade de caminhada em idosos diabéticos e nõo-diabéticos
}

\author{
Gait velocity in diabetic and non diabetic elderly adults
}

\author{
Luciana Pinto de Oliveira Gomes; Fernanda Guimarães Borges'; Izabela Santos Ranconel; \\ Cláudia Santos Oliveira²; Daniela Maria da Cruz dos Anjos ${ }^{3}$ \\ ${ }^{1}$ Fisioterapeutas, graduadas - UNI-BH \\ ${ }^{2}$ Professora do Programa de Mestrado em Ciências da Reabilitação - Uninove - SP \\ ${ }^{3}$ Mestre em Engenharia Biomédica e professora - UNI-BH - Faculdade Pitágoras. \\ ENDEREÇO PARA CORRESPONDÊNCIA \\ Daniela Maria da Cruz dos Anjos \\ R. Julio Pereira da Silva, 510 apto 301 - Cidade Nova \\ 31170-360 - Belo Horizonte - MG [Brasil] \\ E-mail: \\ danianjos@terra.com.br
}

\begin{abstract}
RESUMO
A diabetes mellitus está associada a vários comprometimentos neuromusculoesqueléticos que podem levar a incapacidades físicas e alterações da marcha, como diminuição da velocidade de caminhada, passos curtos, mobilidade de joelho e tornozelo limitada. O objetivo principal deste estudo foi comparar a velocidade da marcha em idosos diabéticos e não-diabéticos por meio do teste de velocidade de 10 metros. Os dados foram analisados por intermédio de Mann-Whitney Test. A pesquisa foi realizada na Clínica Escola do UNI-BH e no Centro de Saúde São Francisco. Nossa amostra foi composta de 47 participantes - 35 mulheres e 12 homens, com idades entre 60 e 75 anos -, dos quais, 24 eram não-diabéticos (grupo 1), e 23, diabéticos (grupo 2). Os resultados deste trabalho mostram que os idosos diabéticos (mediana $1,11 \mathrm{~m} / \mathrm{s}$ ) caminham mais lentamente que os não-diabéticos (mediana $0,95 \mathrm{~m} / \mathrm{s}$ ). Portanto, nossos estudos indicam que idosos diabéticos podem ter um maior declínio funcional quando comparados aos não-diabéticos.
\end{abstract}

Descritores: Diabetes; Idosos; Velocidade de caminhada.

\begin{abstract}
Diabetes Mellitus is related to several neuromuscular skeletal problems that can lead to physical disablement and changes in gait such as reduction of walking speed, short paces and limited mobility of knee and ankle. The main aim of this study is to compare the gait velocity of diabetic and non-diabetic elderly adults using the 10 meters walking test. The data were analyzed using MannWhitney Test. The research was executed at the School-Clinic of UNI-BH and at Centro de Saúde São Francisco. The sample was composed by 47 participants, 24 non-diabetics (group 1), and 23 diabetics (group 2), 35 women and 12 men, all of them aged 60 to 75 years old. The results of the study show that diabetic elderly (median $1,11 \mathrm{~m} / \mathrm{s}$ ) walk more slowly than non-diabetic elderly (median $0,95 \mathrm{~m} / \mathrm{s}$ ). Therefore, it indicates that diabetic elderly can suffer a greater functional decline when compared to non-diabetic elderly.
\end{abstract}

Key words: Diabetes; Elderly; Gait speed. 


\section{INTRODUÇÃO}

No Brasil, a população com idade superior ou igual a 60 anos chega a quase 15 milhões de pessoas ( $8,6 \%$ da população brasileira) e poderá ultrapassar os 30 milhões nos próximos 20 anos - aproximadamente $13 \%$ da população total. Esse aumento da expectativa de vida vem acompanhado, no Brasil, pelas modificações no perfil de saúde da população, com predomínio de doenças crônicas, que geram incapacidades e maiores gastos para o sistema de saúde ${ }^{1,2}$.

Um grande problema associado à velhice é a presença de doenças crônicas degenerativas. Uma das mais prevalentes é a diabetes mellitus (DM), associada com várias comorbidades (vasculares e neuropáticas) que limitam/comprometem ainda mais a funcionalidade e a qualidade de vida do idoso $^{2,3}$. Atualmente, essa doença representa um importante problema de saúde pública, sendo uma das principais síndromes de evolução crônica, com alta morbidade, mortalidade e repercussões econômicas significativas ${ }^{4}$.

Cerca de 20 a $50 \%$ das pessoas com diabetes podem apresentar déficits significantes de sentido de vibração, propriocepção, cinestesia e sensibilidade tátil ${ }^{5}$. A perda de sensibilidade tátil está relacionada com o tempo de diagnóstico ${ }^{6}$ e constitui uma das principais complicações da DM, pois, associada às outras comorbidades da diabetes, contribui para a deteriorização do equilíbrio, alterações dos padrões de caminhada e aumento do risco de quedas ${ }^{5}$. As quedas podem levar o idoso à dependência funcional, além de representar uma das principais causas de morte dessa população ${ }^{7}$.

Além disso, a diabetes está associada a vários comprometimentos neuromusculoesqueléticos e à baixa qualidade de vida auto-relatada ${ }^{8,9,10}$. As incapacidades físicas decorrentes dessa doença podem acarretar alterações da marcha, tais como diminuição da velocidade de caminhada, passos curtos, mobilidade limitada de joelhos e tornozelos, força de flexão plantar do tornozelo diminuída e ativação prematura do tríceps sural, além de provocar alterações na visão, na propriocepção e no equilíbrio ${ }^{11}$. Entre essas alterações, é importante analisar a velocidade de caminhada, pois é uma grande preditora de incapacidades e mortalidades futuras em

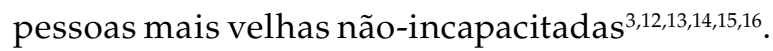
Trata-se de uma medida simples de avaliação do desempenho físico que pode ser usada como teste de seleção para comprometimento da mobilidade ${ }^{17}$. As alterações sensoriais, no tocante à acuidade visual, à sensibilidade plantar e à obesidade, podem influenciar na inabilidade durante a marcha ${ }^{16,18}$.

O idoso tem velocidade de caminhada menor que o adulto jovem ${ }^{14}$. Entre 65 e 90 anos de idade, diminui $10 \%$ por década. Este fato parece estar associado, principalmente, à diminuição do tamanho do passo com o envelhecimento ${ }^{19}$. Ao comparar idosos e jovens que deambulam com a mesma velocidade, verifica-se que os idosos dão passos menores com mais freqüência para desenvolver a mesma velocidade ${ }^{20}$.

Indivíduos com diabetes apresentam velocidade de marcha mais lenta, com uma cadência aumentada e uma circundução durante a fase de balanço ${ }^{21}$. Torna-se necessário verificar o desempenho dessa velocidade em idosos diabéticos e compará-lo com os de não-diabéticos.

O principal objetivo deste estudo foi comparar a velocidade de marcha dos idosos diabéticos com os dos não-diabéticos, utilizando o teste de caminhada de 10 metros, além de correlacionar a velocidade de caminhada com o tempo de diagnóstico, como o índice de massa corporal (IMC), e o número de passos dados durante o teste de caminhada de 10 metros.

\section{MATERIAIS E MÉTODOS}

Trata-se de um estudo transversal, realizado na Clínica Escola do Centro Universitário de Belo Horizonte UNI-BH e no Centro de Saúde São Francisco, em Belo Horizonte. A amostra envolveu 47 participantes - 35 mulheres e 12 homens, com idades entre 60 e 75 anos que vivem em comunidade - dos quais, 23 com história de diabetes e 24 sem história de diabetes. 
Os critérios de inclusão para a seleção foram: idosos diabéticos, classificados clinicamente como tipo 2; idade entre 60 e 75 anos; homens e mulheres; marcha independente, sem ajuda ou em uso de dispositivos auxiliares, e os exclusão: indivíduos com amputação de MMII (membros inferiores); presença de úlceras plantares ativas; doença de Parkinson e neurológicas, exceto neuropatia diabética ou qualquer outra patologia ou condição de saúde que pudesse afetar a marcha desse indivíduos. Foram excluídas também pessoas que apresentassem níveis glicêmicos $\leq 90 \mathrm{mg} / \mathrm{dl}$ e $\geq 300 \mathrm{mg} / \mathrm{dl}$ e PA $\geq 200 / 115 \mathrm{mmHg}$. Nessas condições, a American College Of Sports Medicine (ACSM) ${ }^{22}$ não recomenda que o indivíduo realize teste de esforço ou atividade física, apesar de não se tratar desse tipo de teste.

Após a aprovação do estudo ( $\left.n^{\circ} 054 / 2006\right)$, pelo Comitê de Ética do (UNI-BH) Centro Universitário de Belo Horizonte, os participantes, contatados por telefone ou abordados no local, passaram por triagem na Clínica Escola do UNI-BH e no Centro de Saúde São Francisco. Os voluntários se apresentaram no dia do exame, utilizando roupas leves e sapatos de caminhada habitual. Em todos os participantes, diabéticos ou não, foram aplicados os mesmos testes.

Em uma primeira avaliação, que consistia em coleta de dados pessoais e anamnese (nome, idade, sexo, tipo de diabetes, tempo de diagnóstico, tabagismo, etilismo, dislipidemia e patologias associadas), foi calculado o IMC; em seguida, avaliou-se a sensibilidade tátil e aplicou-se um teste de velocidade de caminhada de 10 metros $^{12,17}$.

A avaliação de sensibilidade tátil foi realizada em um ambiente calmo e sem barulho. $\mathrm{O}$ instrumento utilizado foi o monofilamento Semmes-Weinstein (SW) de 10g, correspondendo ao filamento 5.07. Inicialmente, foi aplicado na fronte do paciente para que ele soubesse $o$ que estava sendo testado. O paciente teve os olhos vendados para não visualizar a aplicação do monofilamento. Os locais testados foram primeiro, terceiro e quinto dígitos plantares; primeira, terceira e quinta cabeça dos metatarsos plantares, centro do médio pé e calcâneo ${ }^{23}$. Se o indivíduo testado fosse incapaz de perceber o monofilamento em quatro pontos ou mais, obtinham-se os resultados de alterações de sensibilidade. O monofilamento SW perdeu sua calibração após uso repetitivo e, em conseqüência disso, pôde-se subestimar a incidência da perda da sensação protetora. Para controlar essa limitação, o monofilamento foi deixado em restauração por 24 horas, após a aplicação em dez pessoas ${ }^{24}$.

O Teste de Caminhada de 10 metros foi utilizado por ser rápido e fácil de administrar e não requerer equipamentos específicos. Os resultados são objetivos e fáceis de registrar ${ }^{12,17}$. Além disso, as confiabilidades intra-avaliador, interavaliador e teste-reteste são elevadas (ICC $=0.9)^{14}$.

O local de realização do teste consistia de um corredor de 10 metros com uma superfície plana, regular e não escorregadia, em ambiente tranqüilo, sem estímulos visuais e auditivos e com boa iluminação.

A velocidade de caminhada foi mensurada em segundos, com um cronômetro, enquanto o sujeito caminhava 8 metros centrais. Um corredor de 10 metros foi marcado com fita adesiva, onde foram demarcados o primeiro e o último metro, considerados como fase de aceleração e desaceleração, respectivamente, e desconsiderados durante a cronometragem. Os participantes foram instruídos a "caminhar a uma velocidade de passo confortável, sem correr" e iniciavam o teste com a palavra "Vai". As instruções para realização do teste foram demonstradas pelas responsáveis do estudo antes de darem início a cronometragem. O escore final era o tempo, em segundos, que o participante levava para deambular 8 metros $^{12,17}$.

O número de passos foi contado durante o teste.

Em todos os testes estatísticos utilizados, foi considerado um nível de significância de 5\%. Para verificar se havia associação entre duas variáveis quantitativas, utilizou-se a correlação de Spearman. 
Para verificar se existe associação significativa entre variáveis categorizadas e quantitativas foi utilizado o teste de Mann-Whitney.

\section{RESULTADOS}

Foram avaliados 47 sujeitos, divididos em dois grupos: o primeiro, composto por 24 idosos não-diabéticos, e o segundo, por 23 idosos diabéticos tipo 2. A caracterização descritiva do perfil dos grupos estudados é apresentada na Tabela 1.

Foi analisada a semelhança entre os grupos 1 e 2 quanto ao gênero, idade e IMC. Verificou-se que havia alguma similaridade entre eles quanto ao gênero e IMC; entretanto, constatou-se diferença estatisticamente significativa em relação à idade $(\mathrm{p}=0,013)$.

No teste de sensibilidade tátil com monofilamentos, os $24(100 \%)$ sujeitos do grupo 1 apresentaram-na tátil preservada, enquanto no 2 , apenas $2(8,7 \%)$ sujeitos manifestaram alteração na sensibilidade plantar.

A velocidade de caminhada do grupo 1 variou de 0,87 a $1,84 \mathrm{~m} / \mathrm{s}(1,14 \pm 0,20)$; no 2 , de 0,71 a $1,22 \mathrm{~m} / \mathrm{s}(0,93 \pm 0,13)$. O número de passos durante o teste do grupo 1 oscilou de 11 a 14 passos $(13,08 \pm 1,02)$; no 2 , de 11 a 21 passos $(14,48 \pm 1,90)$.

Não existe correlação significativa entre a velocidade de marcha e o tempo de diagnóstico (grupo 2) e velocidade de marcha e IMC (grupo 1 e 2).

Foi verificada correlação significativa inversa entre o número de passos e a velocidade de marcha nos dois grupos - grupo $1(\mathrm{r}=-0,352$, $\mathrm{p}=0,051)$ e $2(\mathrm{r}=-0,617, \mathrm{p}=0,002)$. Isso mostra que quanto menor a velocidade de caminhada, maior o número de passos (Figuras 1 e 2).

Na comparação da velocidade de caminhada entre os grupos 1 e 2, foi verificado a existência de diferença estatisticamente significativa $(\mathrm{p}=0,03)$. Observou-se, assim, que o grupo 2 teve uma velocidade de caminhada mais lenta do que o 1 (Tabela 2).
Tabela l: Estatística descritiva do perfil dos grupos estudados, grupo l, não-diabéticos; grupo 2, diabéticos tipo 2

\begin{tabular}{c|c|c}
\hline \multirow{4}{*}{ Características } & Grupo 1 & Grupo 2 \\
\cline { 2 - 3 } & $\begin{array}{c}\text { Não-diabéti- } \\
\cos (\mathrm{n}=24)\end{array}$ & $\begin{array}{c}\text { Diabéticos } \\
(\mathrm{n}=23)\end{array}$ \\
\hline
\end{tabular}

Idade

\begin{tabular}{c|c|c}
\hline Média & 65,58 & 67,91 \\
\hline Desvio-padrão & 1,58 & 3,80 \\
\hline Min-máx & $60-75$ & $60-75$
\end{tabular}

Sexo (\%)

\begin{tabular}{c|c|c}
\hline $\mathrm{F}$ & $83,3 \%$ & 65,2 \\
\hline $\mathrm{M}$ & $16,7 \%$ & 34,8 \\
\hline \multicolumn{3}{|c}{ IMC ( $\left.\mathbf{k g} / \mathbf{m}^{\mathbf{2}}\right)$} \\
\hline Média & 24,63 & 26,6 \\
\hline Desvio-padrão & 2,84 & 3,5 \\
\hline Mín-máx & $19-31,48$ & $20,2-37,5$ \\
\hline \multicolumn{3}{c}{ Tempo diagnóstico (anos) }
\end{tabular}

\begin{tabular}{c|c|c}
\hline Média & & 10,2 \\
\hline Desvio-padrão & & 8,16 \\
\hline Mín-máx & & $0,25-32$ \\
\hline
\end{tabular}

Hipertensão (\%)

\begin{tabular}{c|c|c}
\hline Sim & 76 & 92 \\
\hline Não & 20 & 8 \\
\hline \multicolumn{3}{|c}{ Artrose (\%) } \\
\hline Sim & 32 & 36 \\
\hline Não & 64 & 64 \\
\hline
\end{tabular}

\section{Défct. Vestibular (\%)}

\begin{tabular}{c|c|c}
\hline Sim & 8 & 36 \\
\hline Não & 88 & 64 \\
\hline
\end{tabular}

Défct. Visual (\%)

\begin{tabular}{c|c|c}
\hline Sim & 2 & 5 \\
\hline Não & 98 & 95 \\
\hline \multicolumn{3}{|c}{ Etilismo (\%) } \\
\hline Sim & 8 & 20 \\
\hline Não & 88 & 80 \\
\hline
\end{tabular}

Dislipidemia (\%)

\begin{tabular}{c|c|c}
\hline Sim & 4 & 16 \\
\hline Não & 92 & 84 \\
\hline
\end{tabular}


Tabela 2: Comparação da velocidade de marcha entre os grupos 1 e 2

\begin{tabular}{c|c|c|c}
\hline$::::$ & $\mathrm{N}:$ & Mediana: & Valor $\mathrm{p}$ \\
\hline $\begin{array}{c}\text { Velocidade de } \\
\text { marcha }(\mathrm{m} / \mathrm{s})\end{array}$ & $\begin{array}{c}\text { grupo 1: } \\
24:\end{array}$ & 1,11 & 0,003 \\
\hline $\begin{array}{c}\text { Velocidade de } \\
\text { marcha }(\mathrm{m} / \mathrm{s})\end{array}$ & $\begin{array}{c}\text { grupo 2 : } \\
23:\end{array}$ & 0,95 & \\
\hline
\end{tabular}

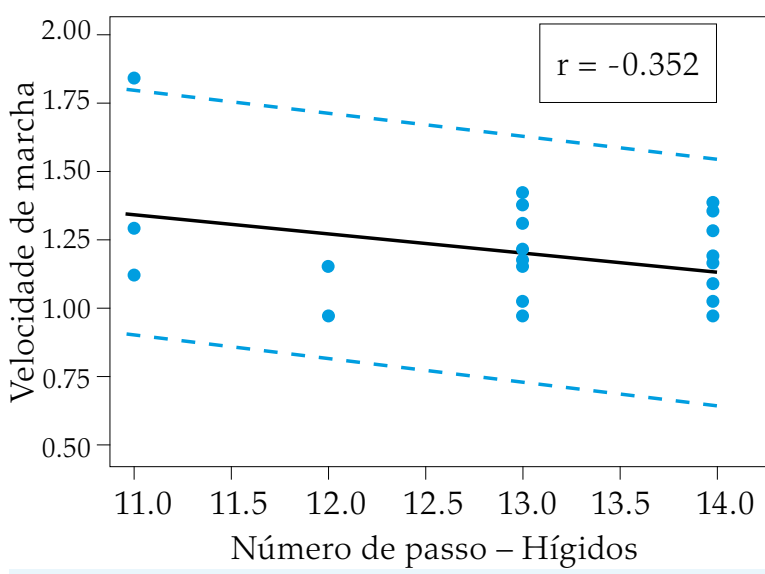

Figura l: Correlação da velocidade de caminhada e o número de passos do grupo 1 $(\mathrm{r}=-0,352, \mathrm{p}=0,051)$

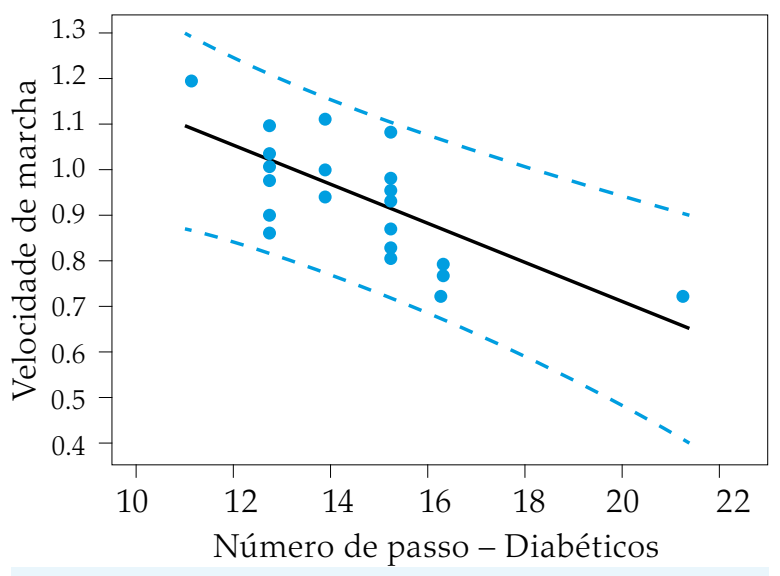

Figura 2: Correlação da velocidade de caminhada e o número de passos do grupo 2 $(\mathrm{r}=-0,617, \mathrm{p}=0,002)$

\section{DISCUSSÃO}

Neste estudo, os idosos diabéticos caminharam mais lentamente que os não-diabéticos. Observou-se também que quanto menor a velocidade de caminhada, maior o número de passos dados pelos sujeitos. O grupo 2 (idosos diabéticos), que caminhou mais lentamente, apresentou maior número de passos.

Esse resultado foi semelhante ao encontrado por $\mathrm{Gregg}^{17}$, em que mulheres idosas diabéticas acima de 60 anos apresentaram uma velocidade de caminhada mais lenta, que a das idosas não-diabéticas.

A principal tarefa motora na marcha envolve o controle motor durante o apoio unipodal, que está diminuído em idosos no decorrer do ciclo da marcha. O período de suporte bipedal é mais estável e altamente preditivo de velocidade da marcha e do comprimento do passo ${ }^{18}$.

Quando se solicita que as pessoas mais velhas aumentem a velocidade da marcha, elas o fazem à custa de maior freqüência das passadas $^{18}$. Modificações adicionais relacionadas com o envelhecimento incluem perda do balanço normal dos braços, diminuição da rotação pélvica e do joelho e aumento da cadência e da altura do passo. Essas modificações da marcha podem representar alterações normais do envelhecimento.

Verificou-se que os dois grupos estudados (idosos diabéticos e não-diabéticos) assemelharam-se no gênero e no IMC; no entanto, apresentaram uma diferença estatisticamente significativa em relação à idade. O grupo dos diabéticos tinha idade média maior, mas parecia haver diminuição na velocidade usual da marcha em diabéticos quando comparados aos controle.

Petrofsky ${ }^{21}$ verificou, em seu estudo, realizado por meio de uma plataforma computadorizada, que, na comparação com o grupo controle, a velocidade de caminhada foi mais lenta e a cadência maior em pacientes diabéticos de uma amostra de pacientes não-idosos composta por homens e mulheres.

Hylton $^{5}$ avaliou sujeitos do sexo feminino e masculino, com diabetes tipo 2 e neuropatia periférica, com idade entre 55 e 91 anos, sendo observado que a velocidade de caminhada e a distância do passo estavam associadas com o sentido de vibração e a sensibilidade tátil dos participantes. 
Nessa pesquisa, a neuropatia diabética não foi avaliada; entretanto, encontraram apenas dois $(8,7 \%)$ diabéticos com alteração de sensibilidade, o que mostra que a alteração de sensibilidade tátil não foi um fator que influenciou a velocidade da marcha nos diabéticos.

As alterações na sensibilidade plantar, assim como a obesidade, podem influenciar na inabilidade durante a marcha ${ }^{16,18}$. Não houve correlação entre o IMC e a velocidade da marcha, mas, na amostra, apenas dois tinham obesidade grau 1. A maioria foi classificada como normal e com sobrepeso.

Yavuzer ${ }^{11}$ comparou diabéticos neuropáticos, não-neuropáticos e grupo controle, não sendo observada diferença significativa da velocidade de marcha entre os diabéticos neuropáticos e não-neuropáticos, como encontrada entre os diabéticos e o grupo controle, o que mostra que a neuropatia periférica não é a única razão para alterações da marcha dos diabéticos.

Alterações músculo-esqueléticas, tais como mobilidade limitada de joelhos e tornozelos, força de flexão plantar do tornozelo diminuída, com a ativação prematura do tríceps sural, alterações da visão, da propriocepção e do equilíbrio encontradas em diabéticos podem influenciar no aumento da velocidade da marcha e na diminuição da cadência ${ }^{11}$.

Courtemanche ${ }^{25}$ e Yavuzer ${ }^{11}$ sustentam a hipótese de que a diminuição na velocidade de caminhada em pacientes com diabetes melittus pode ser uma estratégia compensatória para melhorar a estabilidade durante a marcha.

A marcha é dependente da capacidade de vários órgãos, especificamente os dos sistemas neurológico, músculo-esquelético e cardiovascular. Mais pesquisas avaliando as alterações músculo-esqueléticas foram feitas em decorrência da diabetes, e suas implicações na velocidade da marcha precisam ser avaliadas. Uma melhor compreensão das alterações da marcha pode auxiliar na prevenção das alterações de equilíbrio e das quedas nessa população, além de propor soluções para a perda da capacidade funcional.

\section{CONCLUSÃO}

$\mathrm{Na}$ amostra estudada, foi observado que os idosos diabéticos caminham mais lentamente e executam maior número de passos durante o teste de caminhada de 10 metros. Isso sugere que, na comparação com os idosos não-diabéticos, os diabéticos podem ter maior risco de quedas e de declínio funcional. Mesmo que não haja alteração da sensibilidade, existe mudança na performance da marcha que pode ser atribuída a outras complicações da diabetes, como retinopatia, comprometimento vascular periférico, alterações motoras, proprioceptivas e de equilíbrio.

O aumento da incidência da DM e a maior expectativa de vida dessa população ressaltam a importância de uma atenção especial à avaliação e ao tratamento do desempenho da marcha e do declínio funcional.

\section{REFERÊNCIAS}

1. Menezes RP, Garrido R. O Brasil Está Envelhecendo: Boas e más noticias por uma perspectiva epidemiológica. Rev Bras Psiquiatr. 2002;24(Supl 1):3-6.

2. Instituto Brasileiro de Geografia e Estatística. IBGE. [acesso em 31 de mar. 2008]. Disponível em: www. ibge.gov.br.

3. Gregg EW, Mangione MC, Caurley AJ, Thompson JT, Schwartz VA, Ensrud EK, et al. Diabetes and incidence of functional disability in older Women. Diabetes Care. 2002;1:61-67.

4. Sociedade Brasileira de Diabetes. Consenso brasileiro sobre diabetes 2002: diagnóstico e classificação do diabetes melito e tratamento do diabetes melito tipo 2. Rio de Janeiro: Diagraphic, 2003.

5. Hylton BM, Stephen RL, Richerd CF. Walking stability and sensoriomotor functional in older people with diabetic peripheral neuropathy. Arch Phys Med Rehabil. 2004;85:245-252.

6. Pirart J. Diabetes mellitus and its degenerative complications: a prospective study of 4.400 patients observed between 1974 and 1973. Diabetes Care. 1978;1:168-188. and 252-263.

7. Guimarães NM, Farenatti VTP. Análise descritiva de variáveis teoricamente associadas ao risco de quedas em mulheres idosas. Rev Bras Med Esportes. 2005; 5(11):299-305. 
8. Padua L, Saponara C, Ghirlanda G, Aprile I, Padua $\mathrm{R}$, Pauri F et al. Health-related quality of life in type 1 diabetic patients and influence of peripheral nerve involvement. Neurol Sci. 2001;22:239-45.

9. Resnick HE, Stansberry KB, Haris TM, Tirivedi M, Smith K, Morgan P. Diabetes, peripheral neuropathy, and old age disability. Muscle Nerve. 2002;25:43-50.

10. Arkkila PE, Gautier JF. Musculoskeletal disordens in diabetes mellitus: an update. Best Pract Res Clin Rheumatol. 2002;25:43-50.

11. Yavuzer G, Yetkin I, Toruner F, Koca N, Bolukbas N. Gait deviations of patients with diabetes mellitus: looking beyond peripheral neuropathy. Eura Medicophys. 2006;42:127-133.

12. Odasso MM, Schapira M, Soriano E, Varela M, Kaplan R, Camera L, et al. Gait Velocity as a single predictor of adverse events in healthy seniors aged 75 year and older. Gerontol: Medical Sciences. 2005; $10(60$ a):13041309.

13. Suzuki T, Sugiura M, Furuna T, Nishizawa S, Yoshida H, Ishizaki $\mathrm{T}$, et al. Walking speed as a good predictor for the onset of functional dependence in a Japanese rural community population. Age Aging. 2000;29:441-446.

14. Steffen TM, Hacker TA, Mollinger L. Age and Genderrelated test performance in community-dwelling elderly people: six-minute walk test, berg balance scale, timed get up and go test, and gait speed test. Physical Therapy. 2002;82(8):826-7.

15. Suzuki T, Sugiura M, Furuna T, Nishizawa S, Yoshida $\mathrm{H}$, Ishizaki T, et al. Association of physical performance and falls among the community elderly in Japan in a five year follow-up study. Jpn J Geriatrics. 1999;36:472-478.
16. Gregg E, Beckies G, Williamson D, Leveille S, Langlois J, Engelgau M, et al. Diabetes and Physical Disability Among Older U.S Adults. Diabetes Care. 2006; 23:1272-1277.

17. Odasso MM, Schapira M, Soriano E, Varela M, Kaplan $\mathrm{R}$, Camera L, et al. Gait velocity in senior people. An easy Test for detecting mobility impairment in community elderly. The Journal of Nutrion, healt and aging. 2004; 8(5):340-343.

18. Judge J, Õnpuu S, Davis RB. Effects of age on the Biomechanics and physiology of gait. Clinics In Geriatr Med. 1996;12(4):659-678.

19. Bendall MJ, Bassey EJ. Factors affecting walking speed of elderly people age and aging. 1989;18:327-332.

20. Maciel AC, Guerra RO. Fatores associados à alterações da mobilidade em idosos residentes na comunidade. Rev Bras Fisio. 2005;9(1):17-23.

21. Petrofsky J, Cuneo M, Lee S, Johnson E, Lohman E. Correlation between gait and balance in people with and without type 2 diabetes in normal and subdued light. Med Sci Monit. 2006;12(7):273-281.

22. American College Of Sports Medicine: diretrizes da ACSM para os testes de esforço e sua prescrição; 2003.

23. American Diabetes Association. Consensus development conference on diabetic foot wound care, Diabetes Care. 1999;22:1355-360.

23. Fritschi C. Preventive care of the diabetic foot. Nurs Clin North Am. 2001;36(2):303-20.

24. Courtemanche R, Teasdale N, Boucher P, Fleury M, Lajoie Y, Bard C. Gait problems in diabetic neuropathic patients. Arch Phys Med Rehabil. 1996; 77:849-855. 
